effect of lipid-lowering medication on lipoprotein metabolism in end-stage renal disease.

Carol Lovegrove

Original article Ikewaki K et al. (2005) Delayed in vivo catabolism of intermediate-density lipoprotein and lowdensity lipoprotein in hemodialysis patients as potential cause of premature atherosclerosis. Arterioscler Thromb Vasc Biol [doi: 10.1161/01.ATV.0000188555.60475.c2]

\section{Radiotherapy of left-sided breast cancer does not increase cardiac morbidity}

Radiotherapy following breast-conserving surgery improves overall outcomes and decreases the risk of local recurrence; however, left-sided breast irradiation can cause cardiac damage and might be associated with increased risk of cardiac morbidity. The long-term effects of adjuvant breast irradiation are not well defined, and risk assessment based on early studies might not be valid because modern techniques have reduced the radiation dose and the volume of heart tissue exposed to radiation.

Patt et al. followed women with nonmetastatic breast cancer (8,363 left-sided and 7,907 rightsided) who had received adjuvant radiotherapy between 1986 and 1993. Cox's proportional hazards model was used to compare the incidence of hospitalization caused by ischemic heart disease, valvular heart disease, conduction abnormalities, or cardiomyopathy or heart failure in women with left-sided versus those with rightsided cancer. After a mean follow-up of 9.5 years, there was no significant difference in the rates of hospitalization for cardiac events between women treated with radiotherapy for left-sided and those treated for right-sided breast cancer. The risk of ischemic heart disease increases with time; however, in an additional analysis with 10-15 years of follow-up, there was no difference in left-sided versus right-sided breast cancer.

In summary, the data suggest that modern adjuvant radiotherapy does not cause significant cardiovascular disease. Nevertheless, there is a strong correlation between doses of radiation to the myocardium and risk of death from cardiac events; therefore, additional reductions in myocardial radiation are likely to be beneficial.

Kate Matthews

Original article Patt DA et al.(2005) Cardiac morbidity of adjuvant radiotherapy for breast cancer. J Clin Oncol 23: 7475-7482

\section{Unmasking unexplained cardiac arrest: use of epinephrine and procainamide infusions}

Unexplained cardiac arrest (UCA) in the absence of underlying structural heart disease might be associated with a broad range of differential clinical diagnoses or genetic conditions. Correct diagnosis of the underlying etiology is important not only to determine optimal management, but also for directing genetic testing and possible prophylactic intervention in family members. Although electrocardiography alone may be useful in some patients, long-term monitoring or provocative testing might be required to diagnose latent primary electrical disease.

In this study, 18 patients with UCA but no detectable cardiac disease underwent pharmacologic challenge with epinephrine and procainamide infusion. This technique identified a latent cause for UCA in two-thirds of patients (catecholaminergic polymorphic ventricular tachycardia [CPVT] 56\%, Brugada syndrome $11 \%)$; idiopathic ventricular fibrillation was diagnosed in the remaining $33 \%$ of patients. Of the 55 (8 symptomatic and 47 asymptomatic) family members tested, 9 affected individuals from two families were detected. In all, seven cases of CPVT (five symptomatic and two asymptomatic individuals) and one case of Brugada syndrome (asymptomatic individual) were identified.

Epinephrine and procainamide infusion can be used to unmask the underlying etiology of UCA, helping to diagnose primary electrical diseases such as CPVT and Brugada syndrome. Detection of latent disease in asymptomatic family members using this technique could support genetic testing and might allow early intervention.

Carol Lovegrove

Original article Krahn AD et al. (2005) Diagnosis of unexplained cardiac arrest: role of adrenaline and procainamide infusion. Circulation 112: 2228-2234

\section{Improved myocardial perfusion in transplanted hearts after apheresis treatment}

Cardiac allograft vasculopathy (CAV) is associated with poor outcomes in heart transplant recipients. As elevated levels of 